\title{
Status of the feline retina 5 years after subretinal implantation
}

\author{
Machelle T. Pardue, PhD; ${ }^{1-2 *}$ Sherry L. Ball, PhD; ${ }^{3-4}$ M. Joe Phillips, BS; ${ }^{1}$ Amanda E. Faulkner, BS; ${ }^{1}$ Tiffany \\ A. Walker, BS; ${ }^{1}$ Alan Y. Chow, MD; ${ }^{5}$ Neal S. Peachey, PhD $^{3-4}$ \\ ${ }^{1}$ Atlanta Department of Veterans Affairs Medical Center (VAMC), Decatur, GA; ${ }^{2}$ Department of Ophthalmology, \\ Emory University, Atlanta, GA; ${ }^{3}$ Cleveland VAMC, Cleveland, $\mathrm{OH} ;{ }^{4}$ Cole Eye Institute, Cleveland Clinic Foundation, \\ Cleveland, $\mathrm{OH} ;{ }^{5}$ Optobionics Corporation, Naperville, IL
}

\begin{abstract}
Retinal prosthetics are designed to restore functional vision to patients with photoreceptor degeneration by detecting light and stimulating the retina. Since devices are surgically implanted into the eye, long-term biocompatibility and durability are critical for viable treatment of retinal disease. To extend our previous work, which demonstrated the biocompatibility of a microphotodiode array (MPA) for 10 to 27 months in the normal feline retina, we implanted normal cats with an MPA implant backed with either an iridium oxide or platinum electrode and examined retinal function and biocompatibility for 3 to 5 years. All implants functioned throughout the study period. Retinal function remained steady and normal with a less than 15 percent decrease in electroretinogram response. The retinas had normal laminar structure with no signs of inflammation or rejection in areas adjacent to or distant from the implants. Directly over the implants, a loss of photoreceptor nuclei and remodeling of inner retinal layers existed. These results indicate that the subretinal MPA device is durable and well tolerated by the retina 5 years postimplantation.
\end{abstract}

Key words: biocompatibility, feline, GABA, glutamate, glycine, microphotodiode array, prosthetic, rehabilitation, retina, retinitis pigmentosa.

\section{INTRODUCTION}

The field of retinal prosthetics has greatly expanded since the first report of visual sensations or phosphenes being elicited with electrical stimulation [1-5]. Two main approaches exist: the epiretinal approach in which the implant is placed on the vitreal side of the retina [6] and the subretinal approach in which the implant replaces degenerated photoreceptors [7-8]. The epiretinal approach involves stimulation of the nerve fiber layer with electrodes that receive input from an external camera [6,911]. Alternatively, the subretinal approach activates outer retinal layers with microphotodiodes that respond to incident light in a graded fashion [12-13] or microelectrodes that are externally powered [14-15]. While the various retinal prosthetics depend on different microfabrication technologies and surgical procedures, they share the common objective of stimulating the remaining neural retina in patients with photoreceptor degeneration.

Retinitis pigmentosa (RP) refers to a prevalent class of diseases that involve progressive photoreceptor degeneration. More than a decade of research has led to the realization that RP comprises many distinct disorders involving numerous mutations in a diverse set of genes that are expressed in photoreceptors or retinal pigment

\footnotetext{
Abbreviations: ERG $=$ electroretinogram, FDA $=$ Food and Drug Administration, GABA = gamma-aminobutyric acid, INL = inner nuclear layer, IPL = inner plexiform layer, IrOx = iridium oxide, $\mathrm{MPA}=$ microphotodiode array, $\mathrm{Pt}=$ platinum, $\mathrm{RP}=$ retinitis pigmentosa.

* Address all correspondence to Machelle T. Pardue, PhD; Research Service, 151 Oph, Atlanta VAMC, 1670 Clairmont Rd, Decatur, GA 30033; 404-321-6111, ext 17342; fax: 404-417-2971. Email: mpardue@emory.edu
}

DOI: 10.1682/JRRD.2005.07.0118 
epithelium cells (http://www.retnet.org). This complexity supports the development of a general approach that can be applied to a wide range of disease mechanisms. While growth factors [16-17] or antiapoptotic agents [18] are effective in animal studies and may progress to clinical application, few additional alternatives are in the pipeline. Given this situation, a number of research groups have examined the possibility that a retinal prosthetic capable of transducing light into an electrical signal might restore visual function following photoreceptor degeneration. In fact, preliminary data from clinical trials approved by the U.S. Food and Drug Administration (FDA) suggest improved visual function in patients implanted with subretinal [19] or epiretinal [20] devices. Patients reported both subjective and objective improvements in visual function, including the ability to detect light and motion and recognize objects [19-20]. In addition, some patients implanted with a subretinal device showed improvements in color vision and visual acuity [19].

Even with the successful implantation of retinal prosthetics, some fundamental questions remain about implanting a microelectronic-based device into the ocular cavity for extended periods of time. How long will the implanted components continue to function? Will an immunological reaction be elicited because of the presence of a foreign body? Will the mechanical device maintain a stable position within the eye over time? Will inner retinal changes occur within the eye that may prevent or assist the transmission of visual information generated by the implant? While patient-based studies can provide many answers, animal models will also remain important for defining the long-term biocompatibility of retinal prosthetic devices.

We have previously reported that the microphotodiode array (MPA) device is biocompatible with the feline retina for up to 27 months postimplantation [2123]. While the implant caused a localized loss of photoreceptors directly overlying the area, the inner retinal cells appeared fairly intact with the presence of inner retinal layers of normal thickness [21-23]. Immunocytochemical analysis confirmed that the inner retinal cells adjacent to the implant have normal amino acid neurotransmitter distributions, which suggests normal metabolic activity; inner retinal cells overlying the implant showed a reduction in gamma-aminobutyric acid (GABA) labeling and an increase in glycine labeling that were similar to changes seen with photoreceptor degeneration [23]. Thus, the MPA device is fairly well tolerated by the retina and other ocular structures, with no major indications of glial reactions or other rejection responses [23]. Given that the device is intended for essentially permanent implantation and has been used in FDA-approved clinical trials [19], we have extended our follow-up period.

In this article, we present data from cats that had implants for 3 to 5 years with iridium oxide (IrOx)- and/ or platinum (Pt)-based MPA devices. These data indicate that the subretinal implants remain biocompatible for up to 5 years and also define the amount of time that the current implant design can be expected to operate.

\section{METHODS}

\section{Animal Subjects and Surgical Procedures}

Normal adult cats, maintained on a $12 \mathrm{~h}: 12 \mathrm{~h}$ light:dark cycle and normal cat food, were used in this study. All procedures were approved by the appropriate institutional animal care and use committee and were in accordance with the Association for Research in Vision and Ophthalmology's statement for the use of animals in ophthalmic and vision research.

The vitreoretinal surgical procedure that we used to place the MPA implant in the subretinal space has been described elsewhere [21]. Briefly, using a Zeiss (Thornwood, New York) operating microscope, we made a $3 \mathrm{~mm}$ sclerotomy $\sim 7 \mathrm{~mm}$ posterior to the limbus and then performed a partial vitrectomy. We placed a small saline bubble under the retina to form a localized detachment of the retina from the retinal pigment epithelium and then made a retinotomy at the edge of the saline bleb. We then placed the implant gently in the subretinal space, flattened the bleb by filling the eye with saline, and closed the incisions with sutures.

\section{Implant Design}

MPA devices (Optobionics Corporation, Naperville, Illinois), which are prototype devices similar in structure to artificial silicon retina (ASR ${ }^{\mathrm{TM}}$ ) devices implanted in human trials [19], were produced with crystalline silicon semiconductor fabrication techniques described elsewhere [24]. Briefly, the implant consists of a $2 \mathrm{~mm}$ diameter circular disk with an array of individual $20 \times 20 \mu \mathrm{m}$ photodiode units. In each implant, activated $\mathrm{IrOx}$ or $\mathrm{Pt}$ was used for the electrode layers and titanium was used for the adhesion layer. The implant is powered solely by incident light in the range of 500 to $1,000 \mathrm{~nm}$ with an 
estimated output of $\sim 2 \mathrm{nA} / \mathrm{cm}^{2}$ to $1 \mu \mathrm{A} / \mathrm{cm}^{2}$ under $\sim 100$ lux fluorescent illumination [24].

\section{Fundus Photography}

Fundus photographs were taken with a small-animal hand-held camera (Kowa Optimed, Inc, Torrance, California).

\section{Electroretinography}

After 2 to 3 hours of dark adaptation, electroretinograms (ERGs) were obtained while animals were under sedation (ketamine hydrochloride: $11 \mathrm{mg} / \mathrm{kg}$, xylazine: $2.2 \mathrm{mg} / \mathrm{kg}$ ) and after their pupils had been dilated (1\% mydriacyl, $2.5 \%$ phenylephrine hydrochloride). Responses were recorded with an ERG jet corneal electrode wet with 1 percent methylcellulose. All responses were referenced to a Grass (West Warwick, Rhode Island) gold disk electrode placed in the animal's mouth and grounded with a needle electrode placed subcutaneously in the back. Responses were amplified, filtered $(0.5-1,500 \mathrm{~Hz})$, averaged, and stored with a signal-averaging system (UTAS E-3000, LKC Technologies, Gaithersburg, Maryland).

After each cat was prepared, strobe flash stimuli were presented in an LKC Technologies ganzfeld and dark-adapted ERGs were recorded. Stimulus intensity ranged from -3.4 to $2.1 \mathrm{log} \mathrm{cd} \mathrm{s} / \mathrm{m}^{2}$. For each flash intensity, at least two successive responses were averaged with an appropriate interstimulus interval. A steady adapting field $\left(0.6 \log \mathrm{cd} / \mathrm{m}^{2}\right)$ was presented in the ganzfeld. After 10 min of light adaptation, cone-mediated responses to 25 successive flashes presented at $2.1 \mathrm{~Hz}$ were recorded.

\section{Histology}

We euthanized the cats by anesthetic overdose (pentobarbital: $200 \mathrm{mg} / \mathrm{kg}$ ) and then enucleated the eyes and immersion fixed them in 2.0:2.5 percent paraformaldehyde:glutaraldehyde solution overnight. We rinsed the eyes in $0.1 \mathrm{M}$ phosphate buffer, dissected them to isolate the posterior eyecup, and divided them into $3 \times 2 \mathrm{~mm}$ pieces. Each retinal tissue sample was then embedded in epoxy resin (Embed 812, Electron Microscopy Sciences, Inc, Hatfield, Pennsylvania). We sectioned the embedded retinal pieces at $0.5 \mu \mathrm{m}$ using a histodiamond knife (Diatome, Electron Microscopy Sciences, Inc) on an ultramicrotome and stained them with toluidine blue. The MPA device remained within the subretinal space for all processing, embedding, and sectioning. Measurements of retinal thickness and cell counts were made in $0.5 \mathrm{~mm}$ regions adjacent to the implant, at the edge of the implant, and directly over the implant. Comparable areas of the retinas from the unimplanted eyes were also examined.

To gain additional information about the overall status of the implanted retina, we incubated $0.25 \mu$ m sections overnight with antibodies against one of three of the primary retinal neurotransmitters: GABA, glutamate, or glycine (Abcam PLC, Cambridge, Massachusetts). The sections were rinsed and incubated for 1 hour with $1 \mathrm{~nm}$ gold goat antirabbit immunoglobulin G secondary antibody that was visualized with silver intensification. In addition to the implant sites, we examined areas neighboring the implant sites and corresponding areas in the unimplanted eyes.

\section{RESULTS AND DISCUSSION}

\section{In Vivo Function of Microphotodiode Array Device}

Implant durability is essential in developing a longterm visual prosthetic for patient use. In this study, we tracked implant electrical function using ERG recordings. Figure 1 shows the initial portion of ERGs recorded in response to a $1.88 \log \mathrm{cd} \mathrm{s} / \mathrm{m}^{2}$ ganzfeld flash under lightadapted conditions from three cats examined at 4 and

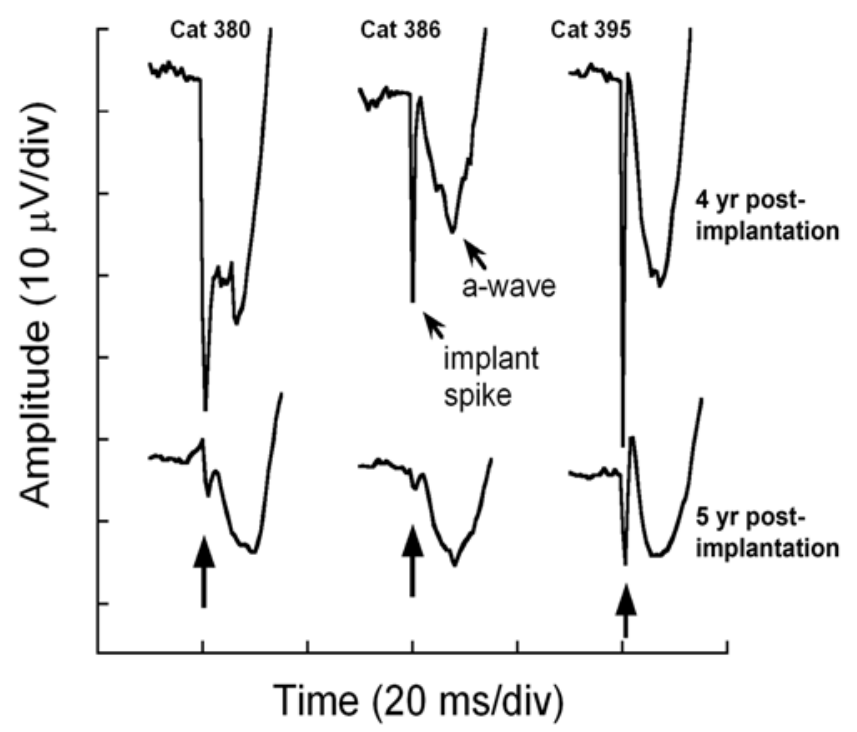

Figure 1.

Average electroretinogram (ERG) a-wave responses from three cats implanted with microphotodiode array device for 4 to 5 years in response to 25 flashes of $1.88 \mathrm{log} \mathrm{cd} \mathrm{s} / \mathrm{m}^{2}$ intensity. Immediately after presentation of flash stimulus, implant response is seen as fast negative spike, followed by negative ERG a-wave. Note that amplitude of implant spike decreases from 4 to 5 years postimplantation. Vertical arrows indicate flash onset. 
5 years postimplantation. These stimulus conditions isolate cone-driven responses that comprise a small negative polarity a-wave followed by a larger positive polarity b-wave. The a-wave has been shown to reflect activity of cone photoreceptors and postreceptoral neurons based on pharmacological studies in primate retina [25]. In each waveform plotted here, a fast negative polarity component that precedes the a-wave is clearly visible. The initial component reflects the electrical response of the MPA device to the strobe flash stimulus since (1) it is not observed in the unimplanted eye (data not shown) and (2) it peaks $\sim 0.5 \mathrm{~ms}$ after flash onset, well before the onset of the cone ERG a-wave [26]. In comparison with the results obtained at 4 years postimplantation, implant spikes obtained 5 years postimplantation were smaller in amplitude for all Pt- and IrOx-based implants, which suggests a possible deterioration of the implant function. Additional studies are needed to determine (1) if the implant continues to function past 5 years and (2) the cause of the implants' decreased spike amplitudes.

Nevertheless, an implant spike was still elicited in each cat tested at 5 years postimplantation, which indicates that both types of implants were still functional at this time. This result suggests that the MPA device provides stimulation to the diseased retina for at least a 5-year period. However, the threshold for therapeutic levels of current are not known for a chronic subretinal device, and thus, we cannot determine from this study whether the current produced by the implant 5 years postimplantation is sufficient to generate visual improvements. One of the long-standing criticisms of the microphotodiode-based device is that the low current output level (nano- to microamperes) will not stimulate existing neurons [13]. Determining with a normal cat model whether the current provided by the MPA device is sufficient to activate overlying retinal neurons is beyond the scope of this study. However, we should note that more recent studies have shown that current levels that produce visual sensations can damage tissue [27] and that low levels of current are sufficient for eliciting visual function [20] and visually evoked cortical responses [28].

\section{Stability of Microphotodiode Array Device in Subretinal Space}

For a subretinal implant, maintenance of a stable position is critical for minimizing mechanical damage to the retina and providing a focused area of stimulation. We used fundus photography to document the location of the implant and assess retinal health. Figure 2 shows fundus photographs of two cats taken at approximately 1 year postimplantation (Figure 2(a) and (c)) and then again at approximately 3 years postimplantation (Figure 2(b) and (d)). In cat 380 (Figure 2(a) and (b)), the large retinotomy created during implantation surgery was still visible over a darkly pigmented area. The magnitude of this area has not changed with time, and we have not observed changes in surgery-associated pigmention in any other cat. The fact that the device remained in a subretinal position is shown clearly by the small retinal vessel that runs across the inferior portion of the retina. In comparison with blood vessels and pigmentary landmarks, we noted the MPA device shifted slightly to a more inferior location. In contrast, cat 388 had a smaller retinotomy and the MPA device remained in a stationary position for the follow-up period (Figure 2(c) and (d)). Fundus photography 5 years postimplantation revealed an additional small shift of the MPA device in cat 380, while the MPA device in cat 388 remained in a stationary position compared with the 3-year data (not shown). Thus, the subretinal implant maintained a fairly stable position over the 5-year follow-up period, which is an important characteristic for a chronic retinal prosthesis. However, in future implantations, creating a smaller retinotomy may allow better positional stability of the implant in the subretinal space.

\section{Retinal Function After Long-Term Implantation}

We used ERGs to assess the overall status of the outer retina. Figure 3 presents representative dark-adapted ERG waveforms from two cats 5 years postimplantation. The responses from implanted eyes and unimplanted eyes are indicated by red and black lines, respectively. Throughout the intensity range examined, the overall ERG waveform was similar between the two eyes of these cats, although responses of implanted eyes were somewhat smaller in amplitude. Across all cats examined with the ERG, the maximum reduction was $\sim 15$ percent. Light-adapted ERGs were also comparable between the implanted and unimplanted eyes (data not shown). Similar changes have been seen at earlier time points [21-22], which indicates that the presence of the implant does not induce a progressive loss of retinal function. In fact, the small reduction in overall retinal function continues to approximately equal the retinal area compromised by the surgical procedures and the implant itself.

\section{Retinal Structure}

Examination of retinal structure is critical in determining long-term biocompatibility of the subretinal 
implant. At all locations away from the implantation and surgical sites, the retina retained a normal appearance (Figure 4). The only changes were found directly overlying the MPA device, where no photoreceptor segments or nuclei were seen. Remodeling of the inner retina layers was seen, including greater disorganization of the inner nuclear layer (INL) and thickening of the Muller cells (Figure 4, arrows). In some cats, the Muller cells appeared to wrap around the edge of the implant (Figure 4(b), triangular arrowhead). Macrophages were also observed directly on the implant surface in cats where the retina was elevated. Note the elevation of the retina over the implant in Figure 4(b) compared with the close retinal location shown in Figure 4(a). The cat retina shown in Figure 4(b) (cat 380) had a large retinotomy that may have allowed vitreous fluid to remain in this location. Fundus photographs from the same cats are shown in Figure 2.

(a)

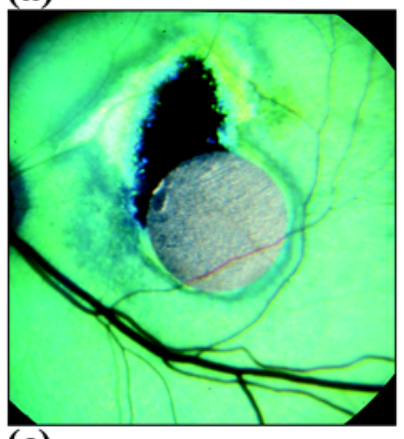

(c)

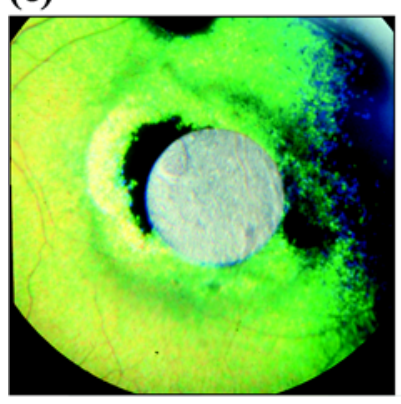

Figure 2.

Fundus photographs of cat 380 at (a) 13 and (b) 34 months postimplantation indicate that implant is completely covered by retina. Surgery-induced pigmentary change appears black through large retinotomy. Implant shifted to slightly inferior location between 13 and 34 months. Fundus photographs of cat 388 at (c) 14 and (d) 37 months postimplantation also show pigmentary changes. Retinotomy is located on left side of implant. Implant remains stable in cat 388.
To determine if cells were lost in areas of the INL over the implant compared with adjacent areas, we made cell counts in five regions: the center of the implant, the two edges of the implant visible in a retinal cross section, and the two areas adjacent to the implant (Figure 5, inset). The average number of cells in the INL of each of these regions is shown in Figure 5. No statistically significant differences between any of the retinal regions examined were noted, and cell counts were comparable with unimplanted eyes. However, a trend toward fewer INL cells overlying the center of the implant existed (mean \pm standard deviation: center $55.5 \pm 16.4$ vs edge $81.9 \pm 19.0$ ).

These results indicated no panretinal rejection or toxicity. The only changes in retinal structure were immediately overlying the implant, as reported previously at earlier postimplantation time points [23]. Although it cannot be confirmed in this study, the slow progressive changes from photoreceptor loss to INL reorganization and cell loss resemble the remodeling described for numerous photoreceptor degenerative conditions [2931]. Thus, the continued remodeling and thinning of the INL may be due to the initial loss of photoreceptors that was caused by the localized retinal detachment from the

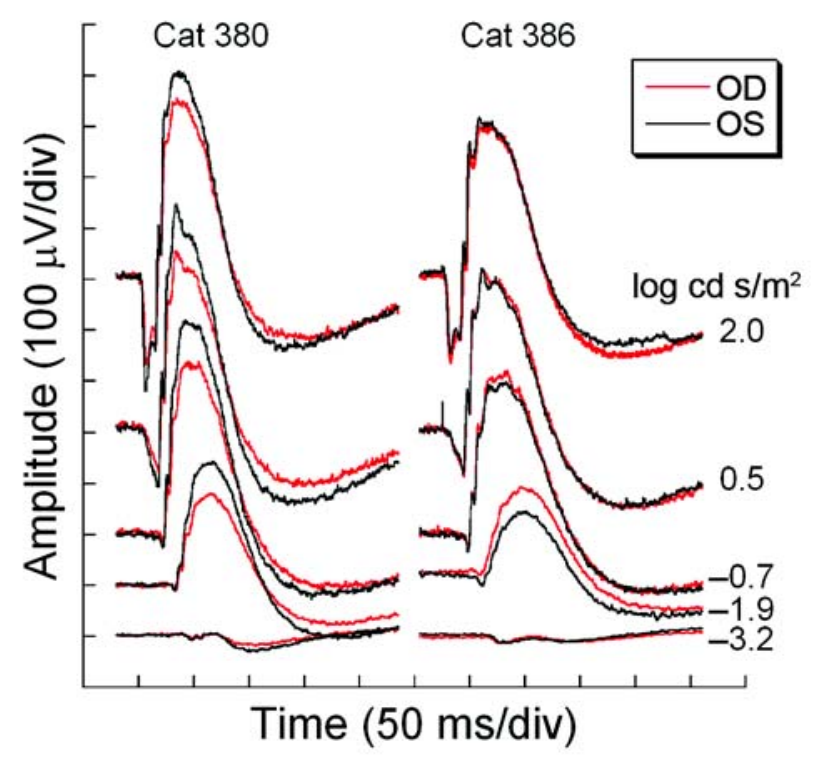

Figure 3.

Full-field dark-adapted electroretinogram waveforms for cats 380 and 386, 5 years postimplantation. Responses were recorded from flash stimuli (-3.2 to $2.0 \log \mathrm{cd} \mathrm{s} / \mathrm{m}^{2}$ ). Each waveform represents an average of 2 to 5 responses from either right implanted eye (OD) (red lines) or left unimplanted eye (OS) (black lines). 
(a)

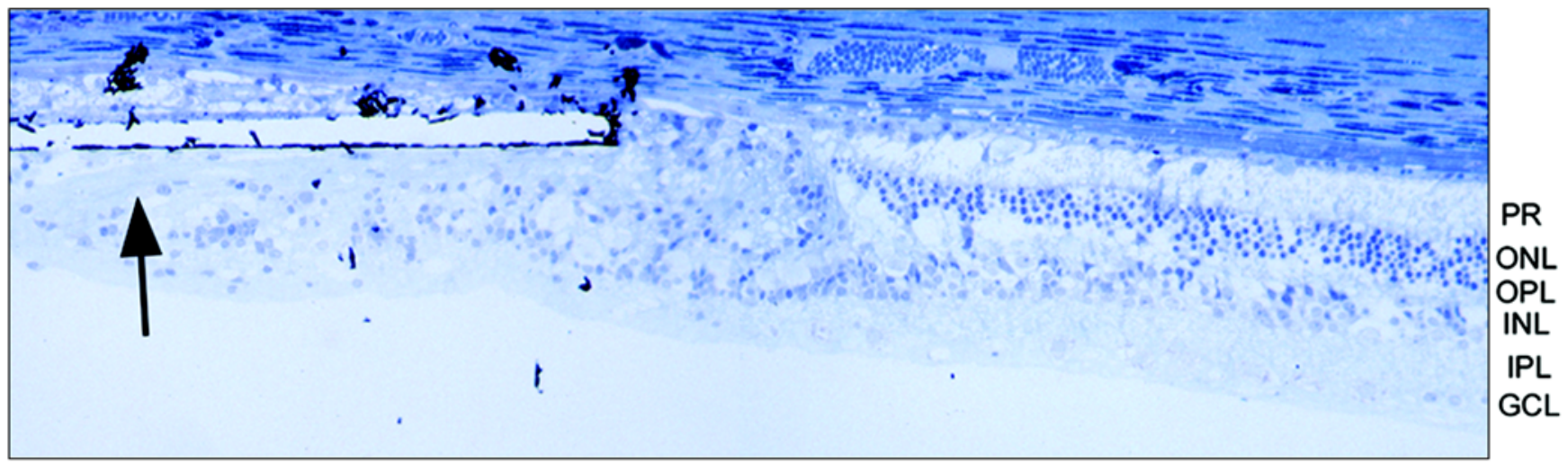

(b)

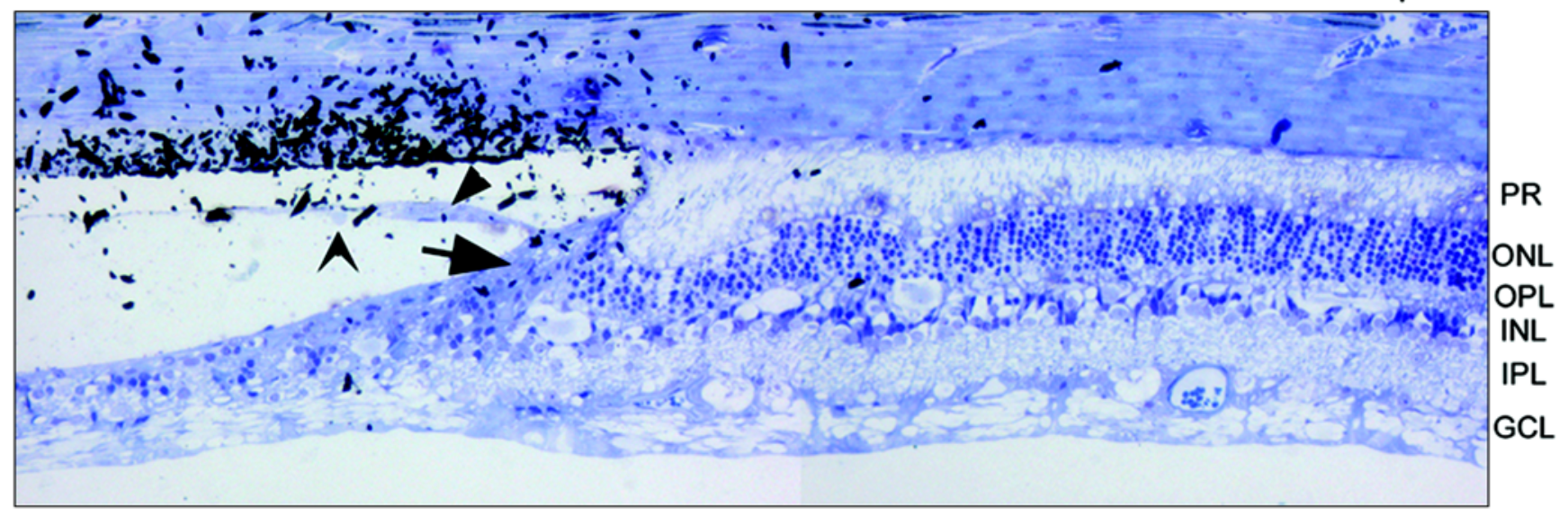

Figure 4.

Light micrographs showing edge of implant in (a) cat 388 and (b) cat 380 after 3 and 5 years of implantation, respectively. Fragments of implant, which were not explanted before histological processing, can be seen as black debris on choroidal side of retina in micrograph. Immediately over implant, retina has degenerated, leaving only disorganized inner retinal layers. This area is likely undergoing remodeling associated with photoreceptor degeneration, which is indicated by formation of glial seal (arrows) formed by Muller cells. (b) Muller cells can be seen to surround edge of implant in some retinas (triangular arrowhead). In addition, macrophages were observed on surface of implant (winged arrowhead) when retina was elevated. Note that retina immediately adjacent to implant had normal appearance. PR $=$ photoreceptors, ONL $=$ outer nuclear layer, OPL = outer plexiform layer, INL = inner nuclear layer, IPL = inner plexiform layer, GCL = ganglion cell layer.

implant, the blockage of nutrients from choroidal circulation from the solid implant, and/or a continued reaction to the presence or activity of the implant. Since the MPA device is designed for patients with RP, the loss of photoreceptors overlying the implant does not exclude the use of this device in these patients. Additionally, studies with the Royal College of Surgeons rat model of RP indicate no changes in the inner retina 8 weeks after implantation [32].

\section{Amino Acid Signature of Retina}

To further characterize retinal metabolic health, we used labeling to assess neurotransmitter patterns after implantation. For each amino acid antibody, distinct changes in labeling patterns were noted in regions directly over the implant. Identification of cell types was made based on the combination of amino acid labeling (the cell's amino acid signature), the location of the cell within the retina, and the shape of the cell body. Figure 6 shows amino acid labeling directly overlying the implant and in an adjacent retinal section. In the normal retina (Figure 6(a)), anti-GABA antibody labels the inner plexiform layer (IPL), the amacrine cells located on the inner lamina of the INL, and the horizontal cells located on the outer lamina of the INL. Directly over the implant, the 


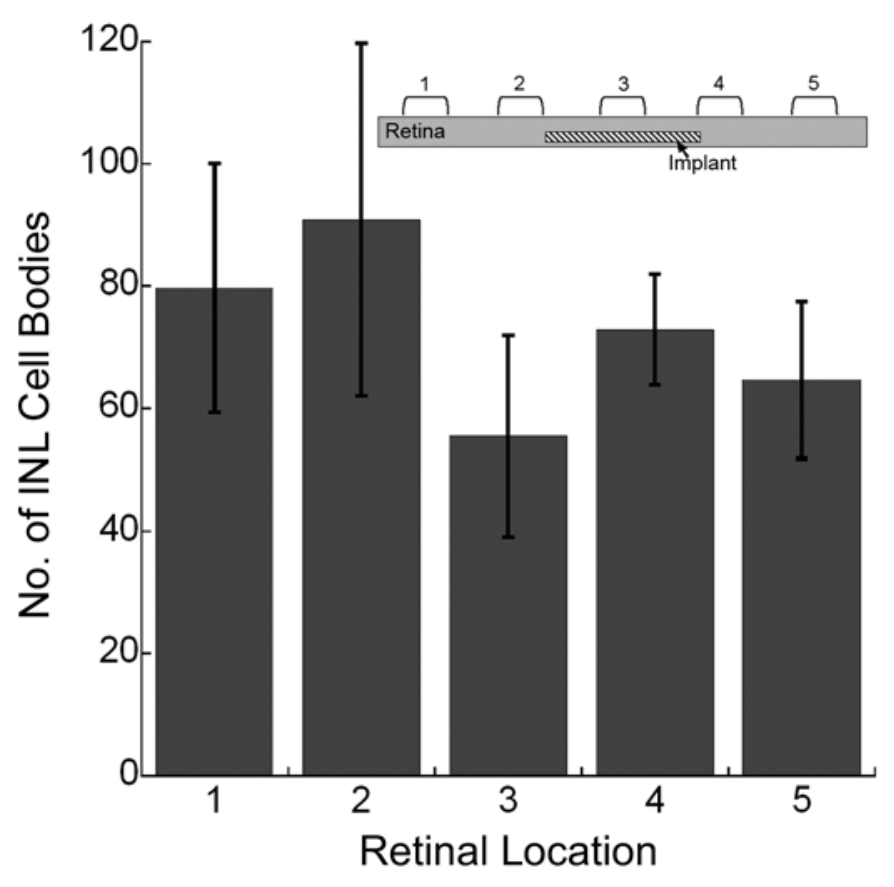

Figure 5.

Average number of nuclei in inner nuclear layer (INL) in area around implant. Inset indicates retinal location where each sample was taken with respect to implant location. No significant reductions were noted in INL nuclei over implant. Error bars represent standard deviation.

labeling was reduced with little or no IPL labeling and scattered INL labeling that appeared to be amacrine cells (Figure 6(b)). In some cats with better preserved inner retinal laminar structure, GABA-labeled horizontal cells could be identified. Antiglutamate labeling (Figure 6(c) and (d)) was dispersed throughout the normal retina (Figure 6(c)) with slightly more concentration in the bipolar cells in the INL and the ganglion cells. Directly over the MPA device (Figure 6(d)), bipolar cells labeled exclusively with glutamate were still visible. However, the bipolar cells had round rather than oval nuclei, which may suggest retraction of dendrites. Antiglycine antibody labeled amacrine cells located in the INL (Figure 6(e)) as well as some bipolar cells in the normal retina. Over the implant (Figure 6(f)), glycine-labeled amacrine cells were present. In all sections from the implantation area, distinct retinal remodeling was evident with all three antibodies as suggested by the disorganization of labeling patterns in the inner retinal nuclei and the absence of labeling in the ganglion cells. The formation of the glial seal was evident as expected from the predicted stages of remodeling following photoreceptor degeneration [29-31], although columns of Muller cell processes were not seen at this stage.

These results show progressive changes in the amino acid labeling patterns in the inner retina compared with our previous study [23] that are similar to those reported for retinal degeneration [29-31]. Thus, these data also support the hypothesis that inner retinal remodeling has occurred, possibly because of the initial loss of photoreceptors.

\section{CONCLUSIONS}

These results suggest that the MPA device was generally well tolerated by the eye, retained a stable position, and continued to function 5 years postimplantation. However, directly over the implant, a loss of photoreceptors and predictable associated secondary changes in the retina existed. Because of the decrease in implant activity over the follow-up period, further studies will be needed to determine if this MPA design will continue to function past 5 years. In addition, clinical trials are in progress to determine visual improvements in patients implanted with subretinal devices of similar design [19], while animal studies are addressing the mechanisms behind these improvements [24,32]. Ultimately, biocompatibility, durability, and efficacy will all be necessary components of a viable visual prosthetic for the treatment of RP.

\section{ACKNOWLEDGMENTS}

This material was based on work supported by the Department of Veterans Affairs, Rehabilitation Research and Development Service (grants C2675CA, C2005R, and C3039R), and Research to Prevent Blindness, New York, New York.

The authors would like to thank Optobionics Corporation, Naperville, Illinois, for providing the retinal prosthetic devices for this study as well as some research support. Dr. Chow performed the surgeries; the study design, data collection, analysis, and interpretation were completed by all the authors; writing and submission of this article were completed by Dr. Pardue with input from Drs. Ball, Chow, and Peachey. Only Drs. Chow and Peachey have financial interest in Optobionics Corporation. 


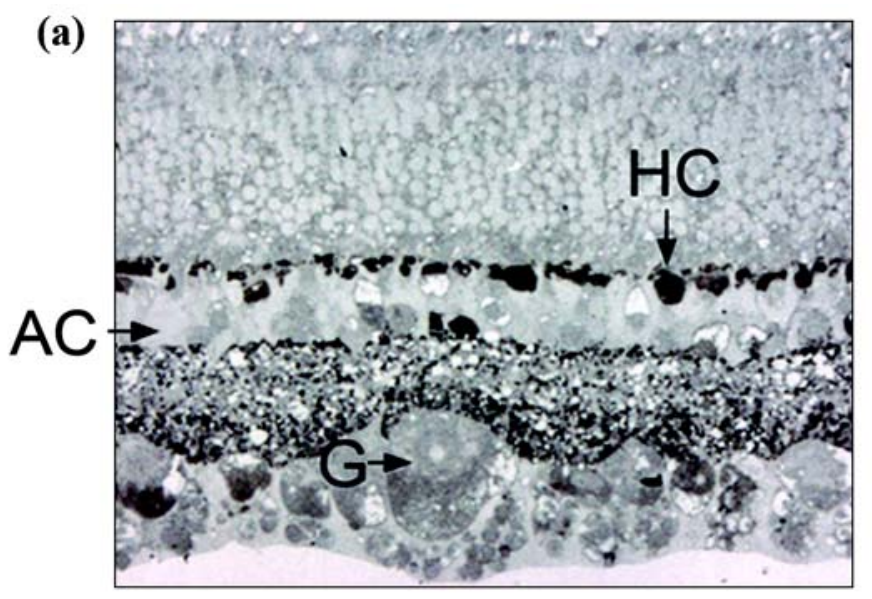

(c)

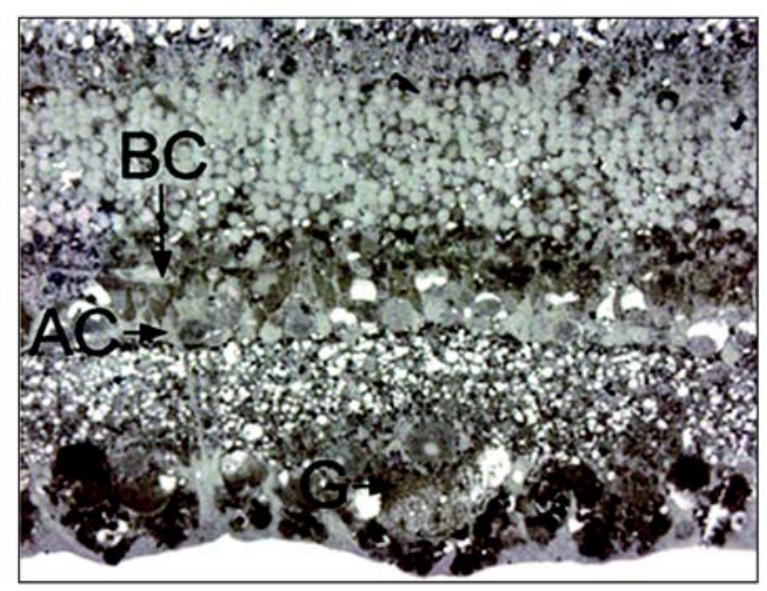

(e)

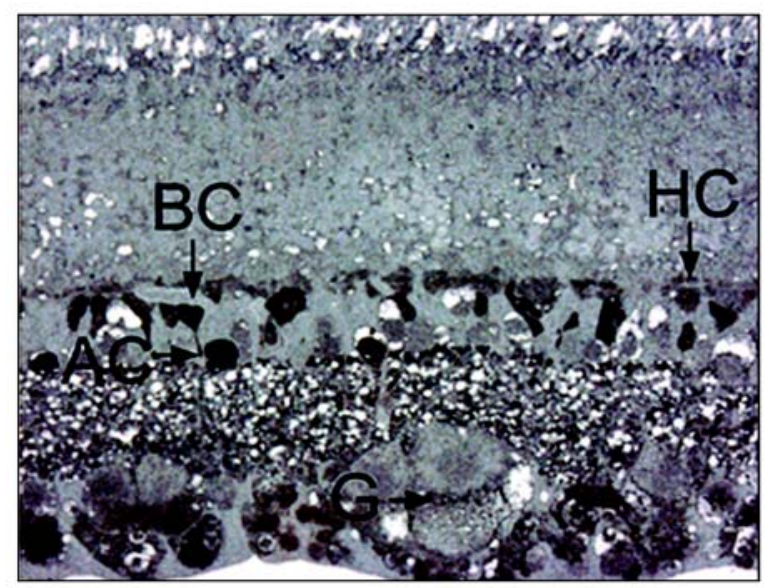

(b)

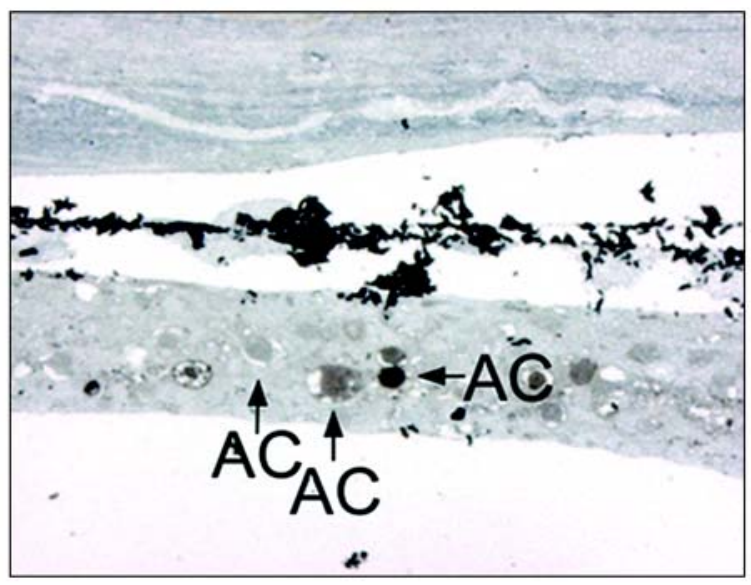

(d)

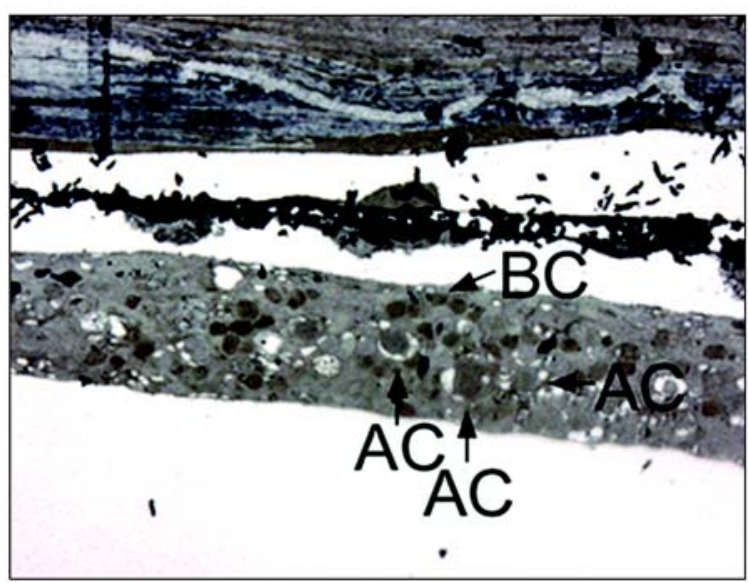

(f)

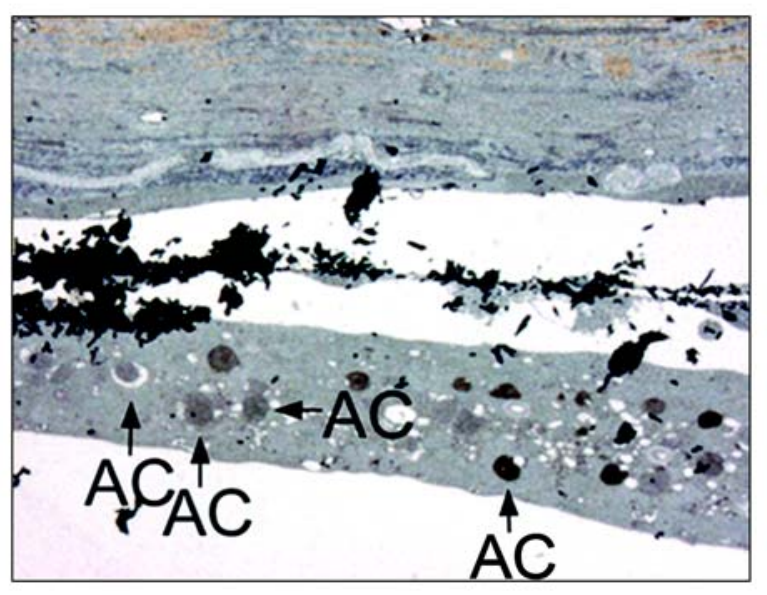

$100 \mu \mathrm{m}$

Figure 6.

Amino acid labeling of cat 386, 5 years postimplantation. Left panels show retina adjacent to implant similar to that seen in unimplanted eye. Right panels show sections of retina overlying implant. Black fragments are remnants of implant after sectioning. Labeling of (a)-(b) anti-GABA, (c)-(d) antiglutamate, and (e)-(f) antiglycine showed normal patterns in adjacent retina and only amacrine and bipolar cells remaining over implant. AC = amacrine cell, $\mathrm{BC}=$ bipolar cell, $\mathrm{HC}=$ horizontal cell, $\mathrm{G}=$ ganglion cell. 


\section{REFERENCES}

1. Brindley GS. The site of electrical excitation of the human eye. J Physiol. 1955;127(1):189-200. [PMID: 14354638]

2. Brindley GS. Beats produced by simultaneous stimulation of the human eye with intermittent light and intermittent or alternating electric current. J Physiol. 1962;164:157-67. [PMID: 14015498]

3. Brindley GS. A new interaction of light and electricity in stimulating the human retina. J Physiol. 1964;171:514-20. [PMID: 14193938]

4. Potts AM, Inuoue J, Buffum D. The electrically evoked response of the visual system (EER). Invest Ophthalmol. 1968;7(3):269-78. [PMID: 5655874]

5. Dawson WW, Radtke ND. The electrical stimulation of the retina by indwelling electrodes. Invest Ophthalmol Vis Sci. 1977;16(3):249-52. [PMID: 844981]

6. Rizzo JF, Wyatt J. Prospects for a visual prosthesis. Neuroscientist. 1997;3(4):251-62.

7. Chow AY, Chow VY. Subretinal electrical stimulation of the rabbit retina. Neurosci Lett. 1997;225(1):13-16. [PMID: 9143006]

8. Zrenner E, Miliczek KD, Gabel VP, Graf HG, Guenther E, Haemmerle H, Hoefflinger B, Kohler K, Nisch W, Schubert M, Stett A, Weiss S. The development of subretinal microphotodiodes for replacement of degenerated photoreceptors. Ophthalmic Res. 1997;29(5):269-80. [PMID: 9323718]

9. Humayun MS, De Juan E Jr, Dagnelie G, Greenberg RJ, Propst H, Philips DH. Visual perception elicited by electrical stimulation of retina in blind humans. Arch Ophthalmol. 1996;114(1):40-46. [PMID: 8540849]

10. Eckmiller R. Learning retina implants with epiretinal contacts. Ophthalmic Res. 1997;29(5):281-89. [PMID: 9323719]

11. Rizzo JF 3rd, Wyatt J, Humayaun M, De Juan E Jr, Liu W, Chow AY, Eckmiller R, Zrenner E, Yagi T, Abrams G. Retinal prosthesis: An encouraging first decade with major challenges ahead. Ophthalmology. 2001;108(1):13-14. [PMID: 11150256]

12. Peyman G, Chow AY, Liang C, Chow VY, Perlman JI, Peachey NS. Subretinal semiconductor microphotodiode array. Ophthalmic Surg Lasers. 1998;29(3):234-41. [PMID: 9547778]

13. Zrenner E. Will retinal implants restore vision? Science. 2002;295(5557):1022-25. [PMID: 11834821] Erratum in: Science. 2002;295(5563):2213.

14. Sachs HG, Schanze T, Wilms M, Rentzos A, Brunner U, Gekeler F, Hesse L. Subretinal implantation and testing of polyimide film electrodes in cats. Graefes Arch Clin Exp Ophthalmol. 2004;243(5):464-68. [PMID: 15578200]

15. Hetling J, Baig-Silva M. Neural prostheses for vision: Designing a functional interface with retinal neurons. Neurol Res. 2004;26(1):21-34. [PMID: 14977054]
16. LaVail MM, Yasumura D, Matthes MT, Lau-Villacorta C, Unoki K, Sung CH, Steinberg RH. Protection of mouse photoreceptors by survival factors in retinal degenerations. Invest Ophthalmol Vis Sci. 1998;39(3):592-602.

[PMID: 9501871]

17. Bok D, Yasumura D, Matthes MT, Ruiz A, Duncan JL, Chappelow AV, Zolutukhin S, Hauswirth W, LaVail MM. Effects of adeno-associated virus-vectored ciliary neurotrophic factor on retinal structure and function in mice with a P216L rds/peripherin mutation. Exp Eye Res. 2002; 74(6):719-35. [PMID: 12126945]

18. Phillips J, Boatright JH, Nickerson JM, Do VT, Pardue MT. Tauroursodeoxycholic acid (TUDCA) preserves photoreceptor function and morphology in rd10 mice at postnatal day 30 [abstract]. Invest Ophthalmol Vis Sci. 2005;46: E-Abstract5237.

19. Chow AY, Chow VY, Packo KH, Pollack JS, Peyman GA, Schuchard R. The artificial silicon retina microchip for the treatment of vision loss from retinitis pigmentosa. Arch Ophthalmol. 2004;122(4):460-69. [PMID: 15078662]

20. Humayun MS, Weiland JD, Fujii GY, Greenberg R, Williamson R, Little J, Mech B, Cimmarusti V, Van Boemel G, Dagnelie G, De Juan E Jr. Visual perception in a blind subject with a chronic microelectronic retinal prosthesis. Vision Res. 2003;43(24):2573-81. [PMID: 13129543]

21. Chow AY, Pardue MT, Chow VY, Peyman GA, Liang C, Perlman JI, Peachey NS. Implantation of silicon chip microphotodiode arrays into the cat subretinal space. IEEE Trans Neural Syst Rehabil Eng. 2001;9(1):86-95. [PMID: 11482368]

22. Chow AY, Pardue MT, Perlman JI, Ball SL, Chow VY, Hetling JR, Peyman GA, Liang C, Stubbs EB Jr, Peachey NS. Subretinal implantation of semiconductor-based photodiodes: Durability of novel implant designs. J Rehabil Res Dev. 2002;39(3):313-21. [PMID: 12173752]

23. Pardue MT, Stubbs EB Jr, Perlman JI, Narftstrom K, Chow AY, Peachey NS. Immunohistochemical studies of the retina following long-term implantation with subretinal microphotodiode arrays. Exp Eye Res. 2001;73(3):333-43. [PMID: 11520108]

24. Pardue MT, Phillips MJ, Yin H, Fernandes A, Cheng Y, Chow AY, Ball SL. Possible sources of neuroprotection following subretinal silicon chip implantation in RCS rats. J Neural Eng. 2005;2(1):S39-47. [PMID: 15876653]

25. Bush RA, Sieving PA. A proximal retinal component in the primate photopic ERG a-wave. Invest Ophthalmol Vis Sci. 1994;35(2):635-45. [PMID: 8113014$]$

26. Friedburg C, Allen CP, Mason PJ, Lamb TD. Contribution of cone photoreceptors and post-receptoral mechanisms to the human photopic electroretinogram. J Physiol. 2004;556(Pt 3): 819-34. [PMID: 14990682] 
27. Vankov A, Huie P, Hakim I, Palanker D. Retinal damage induced by chronic electrical stimulation [abstract]. Invest Ophthalmol Vis Sci. 2005;46:E-Abstract1141.

28. DeMarco PJ, Yarbrough GL, Yee CW, McLean GY, Sagdullaev BT, Ball SL, McCall MA. Stimulaion via a subretinally placed prosthetic elicits central activity and induces a trophic effection visual responses. Invest Ophthalmol Vis Sci. In press 2006.

29. Marc RE, Jones BW, Watt CB, Strettoi E. Neural remodeling in retinal degeneration. Prog Retin Eye Res. 2003; 22(5):607-55. [PMID: 12892644]

30. Marc RE, Jones BW. Retinal remodeling in inherited photoreceptor degenerations. Mol Neurobiol. 2003;28(2):139-47. [PMID: 14576452]
31. Jones BW, Watt CB, Frederick JM, Baehr W, Chen CK, Levine EM, Milam AH, LaVail MM, Marc RE. Retinal remodeling triggered by photoreceptor degenerations. J Comp Neurol. 2003;464(1):1-16. [PMID: 12866125$]$

32. Pardue MT, Phillips MJ, Yin H, Sippy BD, Webb-Wood S, Chow AY, Ball SL. Neuroprotective effect of subretinal implants in the RCS rat. Invest Ophthalmol Vis Sci. 2005; 46(2):674-82. [PMID: 15671299]

Submitted for publication July 1, 2005. Accepted in revised form November 29, 2005. 